\title{
PKD1 negatively regulates cell invasion, migration and proliferation ability of human osteosarcoma
}

\author{
YASUO ONISHI, TERUYA KAWAMOTO, KENTA KISHIMOTO, HITOMI HARA, NAOMASA FUKASE, \\ MITSUNORI TODA, RISA HARADA, MASAHIRO KUROSAKA and TOSHIHIRO AKISUE
}

Department of Orthopaedic Surgery, Kobe University Graduate School of Medicine, 7-5-1 Kusunoki-cho, Chuo-ku, Kobe 650-0017, Japan

Received December 6, 2011; Accepted February 17, 2012

DOI: $10.3892 /$ ijo.2012.1400

\begin{abstract}
Osteosarcoma (OS) is a primary malignancy of the bone, with a tendency to metastasize early. Despite intensive chemotherapy and surgical resection, more than $30 \%$ of patients develop distant metastases, and the prognosis of patients with metastases is essentially poor. Members of the protein kinase D (PKD) family are serine/threonine kinases, and have been studied in various cancers. Among the three different isoforms of this family, PKD1 is one of the best understood for its role in human malignancies; however, its role in musculoskeletal tumors has not been studied. In the present study, we investigated the role of PKD1 in human OS. We first analyzed PKD1 mRNA expression in human musculoskeletal tumor tissue samples by quantitative real-time PCR. PKD1 expression in OS samples was significantly lower than that in benign schwannoma samples, and this was correlated with metastatic potential. In in vitro studies, overexpression of PKD1 by plasmid transfection decreased OS cell invasion, migration and proliferation, and significantly decreased matrix metalloproteinase (MMP)2 mRNA expression. Conversely, siRNA knockdown of PKD1 increased invasion, migration and proliferation of OS cells, and MMP2 expression was markedly increased. Furthermore, overexpression of PKD1 significantly reduced in vivo tumor growth of OS cells. These results demonstrated that low expression of PKD1 may contribute to increased cell invasion, migration and proliferation ability of human OS. Taken together, our findings strongly suggest that PKD1 may negatively regulate the malignant potential of human OS, and may be a therapeutic target for human OS in the clinical setting.
\end{abstract}

\section{Introduction}

Osteosarcoma (OS) is a primary malignancy of bone and has a high propensity for early metastasis, with the lung being the most

Correspondence to: Dr Teruya Kawamoto, Department of Orthopaedic Surgery, Kobe University Graduate School of Medicine, 7-5-1 Kusunoki-cho, Chuo-ku, Kobe 650-0017, Japan

E-mail: trykwmt@med.kobe-u.ac.jp

Key words: PKD1, osteosarcoma, proliferation, invasion, migration, metastasis common metastatic site. Currently, more than $30 \%$ of patients with localized disease eventually develop distant metastases, even after intensive chemotherapy and surgical treatment (1), and despite extensive efforts, the outcome of patients with OS has not significantly improved during the past decade. Molecular mechanisms underlying progression of OS are still largely unknown; therefore, there is a great need to understand the mechanisms of tumor progression before more targeted therapies can be realized.

Protein kinase D (PKD) constitutes a novel family of serine/ threonine kinases with a homology to conventional protein kinase $\mathrm{C}(\mathrm{PKC})$ isoforms in the regulatory domain (2) and diacylglycerol (DAG) receptors, which signal downstream of $G$ protein coupled receptors (GPCRs) and tyrosine kinase receptors. PKD is known to have additional protein modules, including an acidic domain, a hydrophobic domain, and a pleckstrin homology (PH) domain, which are not found in other PKCs, however, their functions have not been fully defined (3). PKD is now classified as a subfamily of the calcium/calmodulin-dependent kinase (CaMK) superfamily because of its unique substrate specificity, which completely differs from other PKCs. PKD is considered to be a key regulator of many important cellular processes (4). The PKD family consists of three isoforms, PKD1/PKC $\mu, \mathrm{PKD} 2$ and PKD3/PKCv, which share unique molecular architecture (3). Among the three different isoforms, PKD1 is the most extensively characterized. It is a target of DAG and a direct substrate of PKCs, and recent studies revealed that PKD1 has important functions in the regulation of cancer cell adhesion, vesicle transport, survival, migration, invasion, proliferation and apoptosis (5-8). A potential mechanism of PKD1 regulation of cell survival is via activation of the nuclear factor (NF) $-\kappa B$ pathway (8). PKD1 has been implicated in the regulation of a variety of cellular functions, including DNA synthesis, cell proliferation (9), cell migration (10), and NF- $\kappa \mathrm{B}-$ mediated gene expression. However, recent studies demonstrated that PKD1 acts as a negative regulator in several cancer cells $(11,12)$. PKD1 inhibits cell migration of prostate cancer cells (11), and in breast cancer cells, PKD1 negatively regulates cell motility via its activation by the RhoGTPase RhoA, which has also been implicated in the inhibition of cell migration (13). Expression of PKD1 is downregulated in androgen-independent prostate cancer (14), and in gastric cancer its promoter is epigenetically-silenced by methylation events (15). PKD1 has been also reported as a regulator of the 
matrix metalloproteinase (MMP) expression and inhibits breast cancer cell invasion (16). Thus, PKD1 has opposing biological functions depending on the cell type-specific environment; however, the role of PKD1 in OS is not fully understood.

For the development of metastases, tumor cells require invasive capacity in order to degrade the extracellular matrix (ECM) (17), and MMPs are known to degrade most components of ECM (18). In various human malignancies, the increased expression of certain MMPs correlates with tumor expansion, invasiveness and poor prognosis of patients with the disease (19), and among them, MMP2 is associated with tumor invasiveness and metastatic potential in OS (20).

Here, we showed that PKD1 expression was decreased in OS samples compared with benign schwannoma samples, and that its expression in OS samples with metastases was significantly lower than in non-metastatic OS samples. In in vitro studies, we demonstrated that overexpression of PKD1 by plasmid transfection decreased OS cell invasion, migration and proliferation, and conversely, PKD1 inhibition increased these abilities. We also identified PKD1 as an inhibitor of MMP2 expression, which has been implicated in the progression of human OS. In addition, we found that PKD1 overexpression significantly suppressed the in vivo tumor growth of OS cells. Our findings presented in the present study strongly suggest that PKD1 may have negative effects on tumorigenesis and/or tumor progression of human OS, and that PKD1 may be an effective therapeutic target for human OS in the clinical setting.

\section{Materials and methods}

Musculoskeletal tumor tissue samples and human OS cell lines. Twenty-eight human musculoskeletal tumor tissue samples, including 13 osteosarcomas (5 samples with lung metastases) and 15 schwannomas, were obtained by surgery at Kobe University Hospital in accordance with institutional guidelines. All patients participating in this study gave informed consent prior to surgery. All samples were immediately stored at $-80^{\circ} \mathrm{C}$ until use.

Three human OS cell lines, KHOS, KTHOS and MG63 were used in the in vitro studies. KHOS and MG63 cells were obtained from the American Type Culture Collection (ATCC), and the KTHOS cell line was previously established in our laboratory (21). Cells were grown in culture medium consisting of Dulbecco's modified Eagle's medium (DMEM; Sigma-Aldrich Co., St. Louis, MO, USA) supplemented with $10 \%$ fetal bovine serum (FBS; Sigma-Aldrich) and $100 \mathrm{U} / \mathrm{ml}$ penicillin/streptomycin solution (Sigma-Aldrich). Cell lines were routinely maintained at $37^{\circ} \mathrm{C}$ in a humidified $5 \% \mathrm{CO}_{2}$ atmosphere.

Quantitative real-time PCR. We isolated total RNAs from tumor tissue samples and cell lines using an RNeasy Mini Kit according to the manufacturer's protocol (Qiagen, Valencia, CA, USA), and first strand cDNAs were transcribed. Quantitative real-time PCR (qRT-PCR) was performed in a $20-\mu 1$ reaction mixture using the Power SYBR Green Master Mix reagent (Applied Biosystems, Foster City, CA, USA) on an ABI PRISM 7500 sequence detection system (Applied Biosystems). The PCR conditions were as follows: 1 cycle at $95^{\circ} \mathrm{C}$ for $10 \mathrm{~min}$ followed by 40 cycles at $95^{\circ} \mathrm{C}$ for $15 \mathrm{sec}$ and $60^{\circ} \mathrm{C}$ for $1 \mathrm{~min}$. Primers used were: $P K D 1,5$ '-AATTCCTAATGGGGCCAATC-3' and 5'-TGA CCACATTTTCTCCCACA-3'; $M M P 2,5$ '-ACAGCAGGTCTC AGCCTCAT-3' and 5'-TGCCTCTGGACAACACAGAC-3'; PCNA, 5'-ATGCCTTCTGGTGAATTTGC-3' and 5'-TCACTC CGTCTTTTGCACAG-3'; $\beta$-actin, 5'-GATGAGATTGGCAT GGCTTT-3' and 5'-CACCTTCACCGTTCCAGTTT-3'. The values were normalized with those for $\beta$-actin, and relative expression was analyzed using the $\Delta \Delta \mathrm{C}_{\mathrm{t}}$ method.

Transfection of OS cell lines with PKD1-expressing plasmid or small interfering RNA for PKD1. To evaluate the effect of PKD1 expression, we transfected OS cells with PKD1-expressing plasmid or human PKD1-specific small interfering RNA (siRNA).

For PKD1 overexpression, OS cells were transfected with a PKD1-expressing plasmid, HA.PKD1 or pcDNA3 as control (Addgene, Cambridge, MA, USA). Briefly, 1 day before transfection, cells were seeded in a 6-well culture plate in growth medium without antibiotics. Then, cells were transfected with $2 \mu \mathrm{g}$ of plasmid using FuGENE HD Transfection Reagent according to the manufacturer's protocol (Roche Applied Science, Indianapolis, IN, USA). For PKD1 knockdown, we used a human PKD1-specific siRNA (PKD1-siRNA) and a negative control siRNA (control-siRNA) (Ambion Inc., Austin, TX, USA). Cells seeded in a 6-well culture plate were transfected with 100 pmol of either PKD1-siRNA or control-siRNA using Lipofectamine 2000 Transfection Reagent according to the manufacturer's protocol (Invitrogen, Carlsbad, CA, USA).

Immunoblot analysis. Lysates were extracted from cells using a whole cell lysis buffer (Mammalian Protein Extraction Reagent; Thermo Scientific, Rockford, IL, USA) supplemented with protease and phosphatase inhibitor mix (Roche Applied Science). The protein content of lysates was then quantified using BCA Protein Assay reagent (Bio-Rad, Richmond, CA, USA). Samples containing equal amounts of proteins were electrophoresed through $12 \%$ polyacrylamide gels, and were transferred onto PVDF membranes. After blocking, membranes were incubated overnight at $4^{\circ} \mathrm{C}$ with the following antibodies in CanGet Signal Solution 1 (Toyobo Co., Ltd., Osaka, Japan): anti-human PKD1 polyclonal antibody (1:1000; Cell Signaling Technology Inc., Danvers, MA, USA), anti-human MMP2 polyclonal antibody (1:1000; Cell Signaling Technology) and anti- $\alpha$-tubulin antibody (1:10000, Sigma-Aldrich). Following washes, membranes were incubated with appropriate secondary antibody conjugated to horseradish peroxidase, and were exposed with ECL Plus western blotting detection system reagent (GE Healthcare Bio-Sciences, Piscataway, NJ, USA). Antibody binding was detected by Chemilumino analyzer LAS-3000 mini (Fujifilm, Tokyo, Japan).

Cell invasion assays. In vitro invasive properties of OS cells were evaluated using transwell chamber invasion assays, as previously described (22). After $48 \mathrm{~h}$ of plasmid or siRNA transfection, cells were added to the upper wells of 24-well transwell chambers (BioCoat Matrigel Invasion Chamber; BD Biosciences, Bedford, MA, USA), and lower wells were filled with complete growth medium. The chambers were incubated to allow cells to invade from the upper wells towards the lower wells. After $22 \mathrm{~h}$ of incubation, non-invading cells on the upper surface of membranes 
A

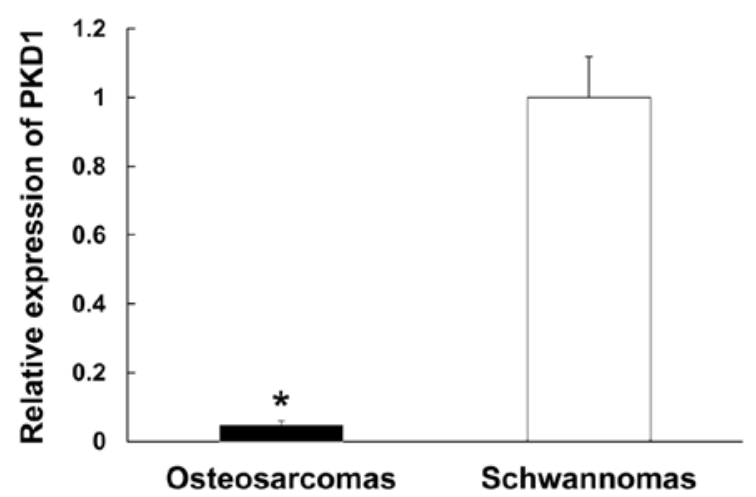

B

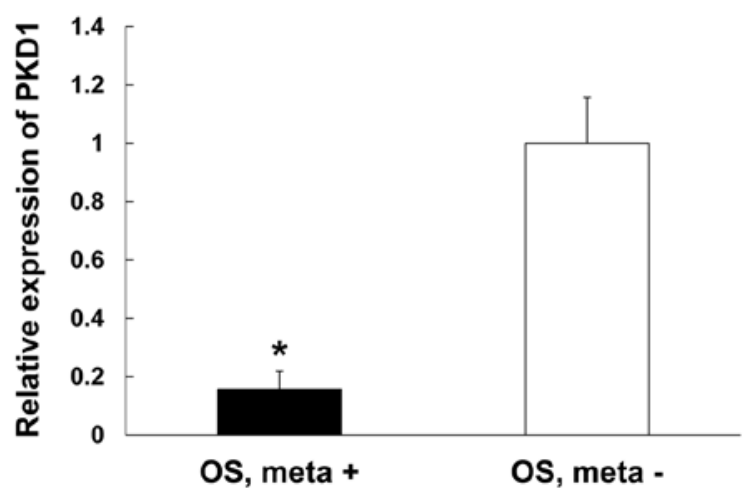

Figure 1. qRT-PCR analysis of PKD1 mRNA expression in human musculoskeletal tumor tissue samples. (A) mRNA expression of PKD1 in osteosarcoma (OS) samples was significantly lower than that in benign schwannoma samples $\left({ }^{*} \mathrm{P}<0.05\right)$. (B) In OS samples with lung metastases, mRNA expression of PKD1 was significantly lower than that in non-metastatic OS samples $\left({ }^{*} \mathrm{P}<0.05\right)$.

were removed by scrubbing, and invading cells on the lower surface of the membranes were fixed and counted under a light microscope in three random fields.

Cell migration assays. We performed in vitro scratch woundhealing assays to evaluate OS cell migration as previously described (23). After $48 \mathrm{~h}$ of transfection, cells were seeded in a 6-well culture plate to form a confluent monolayer. A denuded area was created by scraping with a sterile $200-\mu$ l pipette tip, and each well was washed three times with PBS to remove floating cells. Scratch wounds were then inspected microscopically and photographs of each wound were taken at indicated times ( 0 and $16 \mathrm{~h}$ after scratch). The distance between the opposing edges of wound was measured at two points and averaged.

Cell proliferation assays. We assessed the cell proliferative activity of transfected cells using Cell Counting Kit-8 (CCK-8) according to the manufacturer's instructions (Dojindo Inc., Kumamoto, Japan). Cells that were transfected with plasmid or siRNA were seeded in 96-well culture plates in $100 \mu$ l culture medium. At the indicated times (0,24, 48 and $72 \mathrm{~h}$ of incubation), $10 \mu \mathrm{l}$ of the CCK- 8 solution was added into each well, and the optical density was measured at a wavelength of $450 \mathrm{~nm}$ using a Model 680 Microplate Reader (Bio-Rad, Richmond, CA, USA) after $2 \mathrm{~h}$ of further incubation. The relative number of viable cells in each well was calculated.

Animal models. Finally, we evaluated the effect of PKD1 overexpression on in vivo OS cell proliferation using animal models. Male athymic BALB/c nude mice, aged 6-8 weeks, obtained from CLEA Japan (Tokyo, Japan) were maintained in pathogenfree conditions and in accordance with institutional principles. All animal experiments were performed according to the Guide for the Care and Use of Laboratory Animals at the host institution and were approved by the institutional animal committee. A human OS cell line, MG63, was transfected with either HA.PKD1 or control plasmid as described above. After 3 days of plasmid transfection, cells $\left(4.0 \times 10^{6}\right.$ cells in $100 \mu$ l culture medium) were inoculated subcutaneously to the dorsal area of nude mice (12 mice for each transfection). After cell inoculation, the volume and body weight of mice was monitored twice a week for 28 days. Tumor volume was calculated according to the formula $\mathrm{V}=\pi / 6 \times \mathrm{a}^{2} \mathrm{x} \mathrm{b}$, where $\mathrm{a}$ and $\mathrm{b}$ represent the shorter and longer dimensions of the tumor, respectively (24). After 28 days of inoculation, mice were euthanized, and tumors were excised from the mice. The expression of PKD1 in excised tumors was evaluated by qRT-PCR and immunohistochemical analysis was performed as previously described (24). For immunohistochemical staining, anti-PKD1 antibody (Bioworld Technology Inc., Minneapolis, USA) was used as the primary antibody.

Statistical analysis. Each experiment was performed at least three times independently, and data are presented as the mean \pm SD. For distributed data, the two-tailed t-test was used for a comparison between groups. ANOVA with post hoc test was used to compare for continuous values. All tests were considered significant at $\mathrm{P}<0.05$.

\section{Results}

PKD1 expression was significantly lower in OS samples compared with benign schwannoma samples, and was associated with metastatic potential. First, we surveyed the mRNA expression of PKD1 in musculoskeletal tumor tissue samples by qRT-PCR. We found that PKD1 mRNA expression in OS samples was significantly lower than that in benign schwannoma samples ( $\mathrm{P}<0.05$, Fig. 1A). In addition, low expression of PKD1 showed a significant correlation with lung metastases in OS samples $\left({ }^{*} \mathrm{P}<0.05\right.$, Fig. 1B). These results suggest that low expression of PKD1 contributes to musculoskeletal tumorigenesis, and to the metastatic potential of OS.

PKD1 overexpression decreased OS cell invasion. We performed transfection of PKD1-overexpressing plasmid (HA.PKD1) or PKD1-siRNA to evaluate the effects of PKD1 expression on OS cells. PKD1 expression was sufficiently increased by HA.PKD1 transfection (Fig. 2A) and was reduced by PKD1-siRNA transfection (Fig. 2B) compared with each control.

To examine the effect of PKD1 expression on OS cell invasion, we performed transwell invasion assays. The number of invading cells in HA.PKD1 transfected cells was decreased to 
A
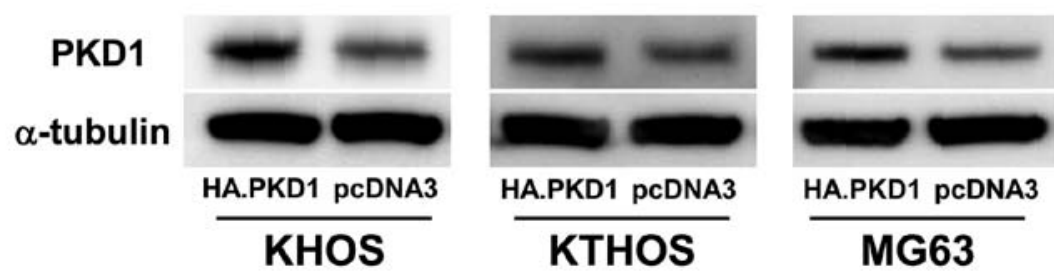

B
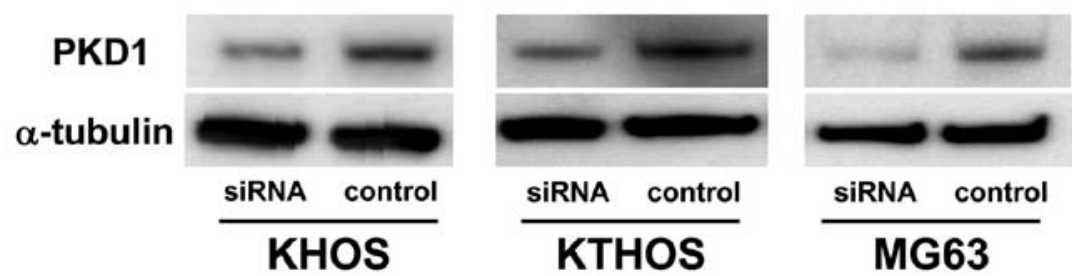

C

KHOS
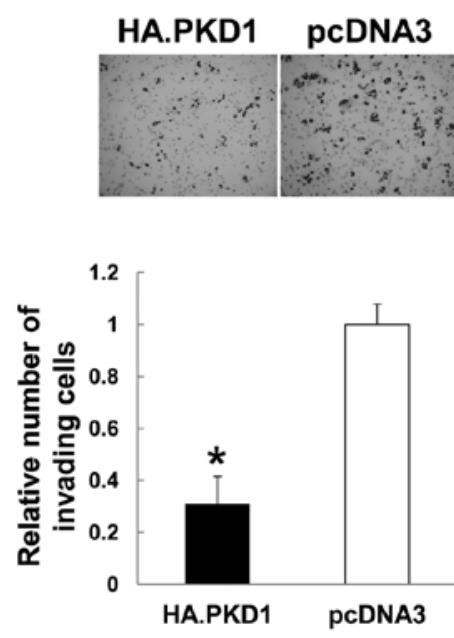

$\mathrm{D}$
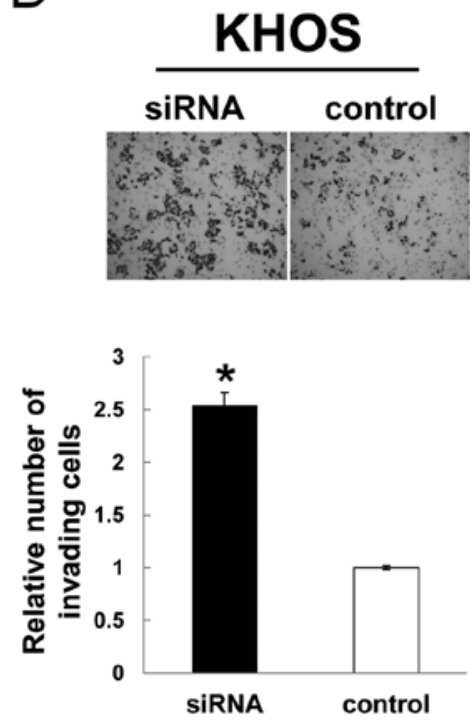

KTHOS
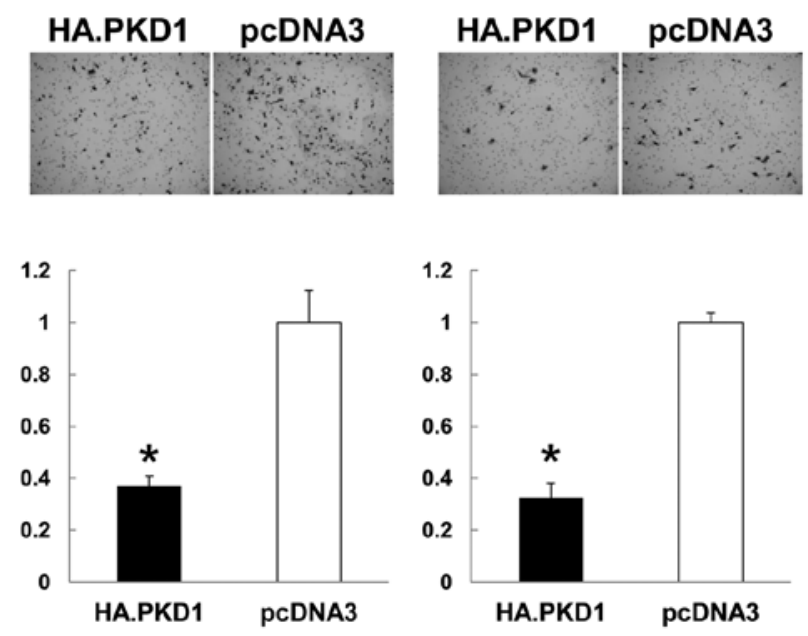

\section{MG63}

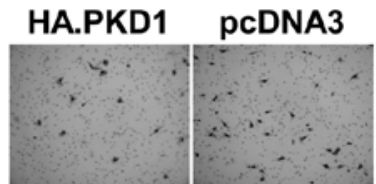

KTHOS
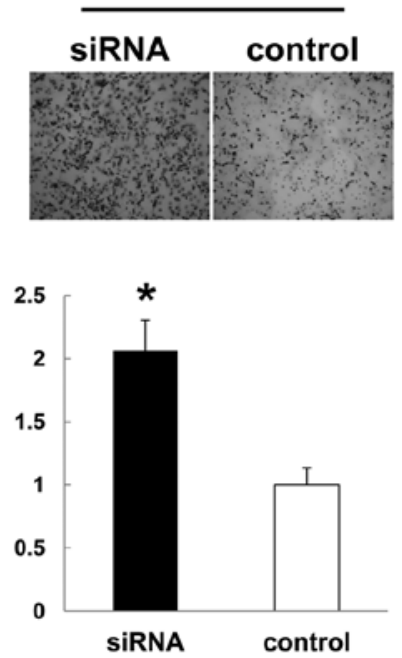

MG63
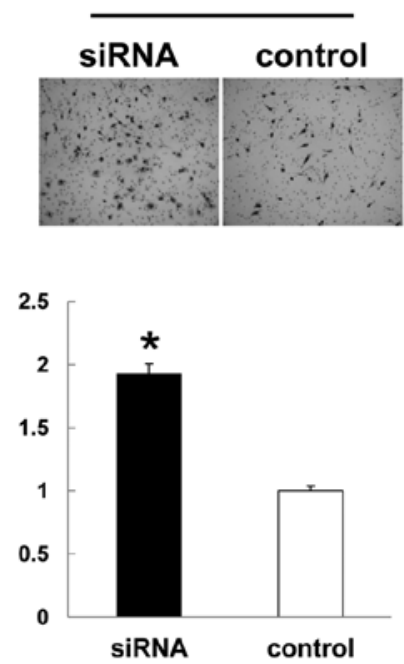

Figure 2. Immunoblot analysis for protein expression of PKD1 in plasmid- or siRNA-transfected OS cells (A and B). We performed transfection of OS cell lines with PKD1-overexpressing plasmid (HA.PKD1) or control plasmid (pcDNA3) (A), and PKD1-siRNA or control siRNA (B). In all three OS cell lines, PKD1 protein expression was increased by HA.PKD1 transfection (A) and was reduced by PKD1-siRNA transfection (B). Transwell chamber invasion assays of plasmid- or siRNA-transfected OS cells (C and D). The number of invading cells in HA.PKD1 transfected cells was decreased to 31 (KHOS), 36 (KTHOS) and 32\% (MG63) of that in control cells (" $\mathrm{P}<0.05)$ (C). Conversely, siRNA knockdown of PKD1 increased the number of invading cells by 253,206 and $191 \%$ of that in control cells, respectively $\left({ }^{*} \mathrm{P}<0.05\right)(\mathrm{D})$. 
A

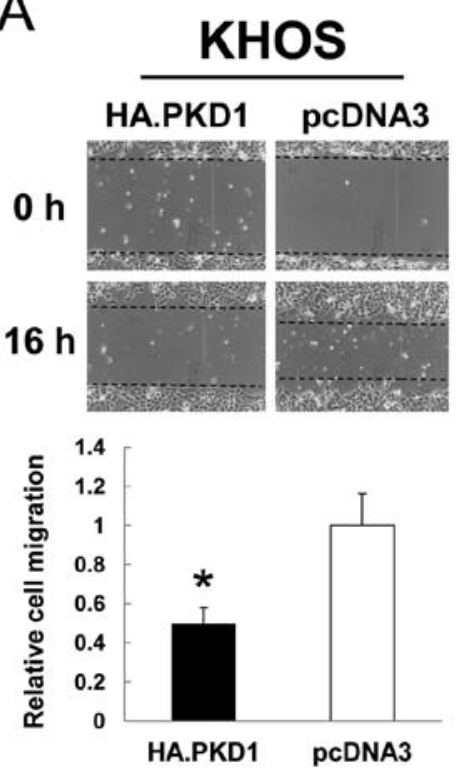

B
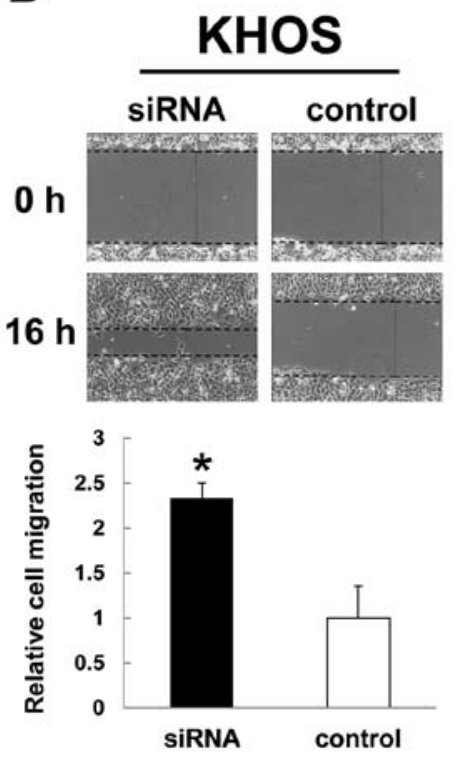

KTHOS
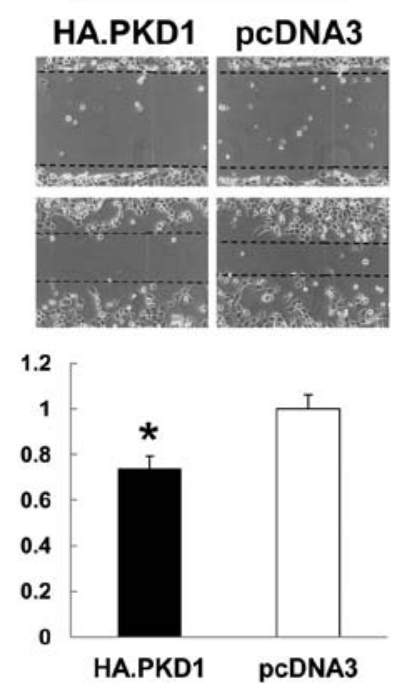

KTHOS
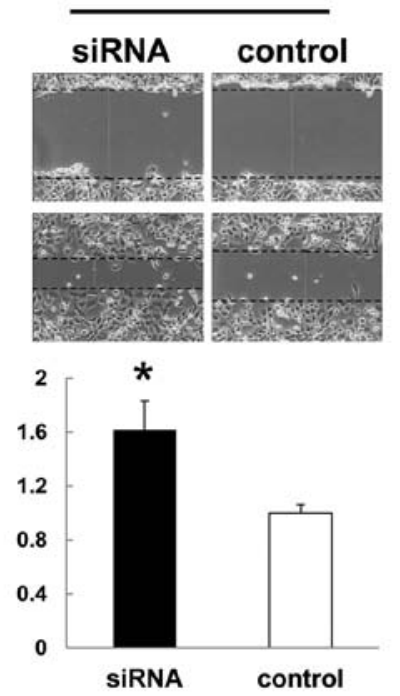

MG63
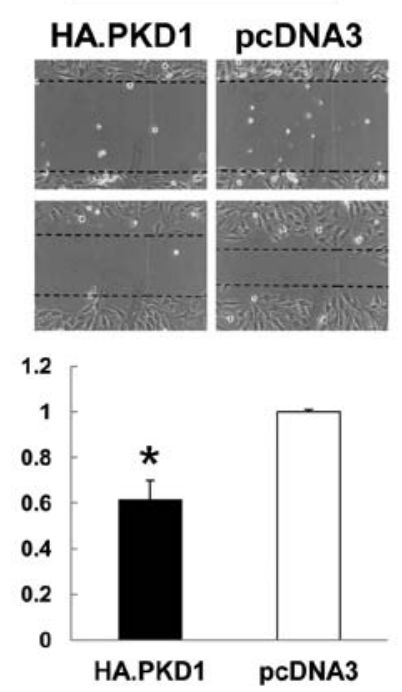

Figure 3. In vitro scratch wound healing assays of plasmid- or siRNA-transfected OS cell lines. PKD1 overexpression significantly inhibited wound closure in all OS cells after $16 \mathrm{~h}$ of incubation $\left({ }^{*} \mathrm{P}<0.05\right)(\mathrm{A})$, and conversely, siRNA knockdown of PKD1 induced a significant increase in wound closure compared with controls $\left({ }^{*} \mathrm{P}<0.05\right)(\mathrm{B})$.

31 (KHOS), 36 (KTHOS) and 32\% (MG63) of that of control cells ( $\mathrm{P}<0.05$, Fig. 2C), and conversely, siRNA knockdown of PKD1 increased OS cell invasion by 253, 206 and 191\% compared with control cells, respectively ( ${ }^{*} \mathrm{P}<0.05$, Fig. $2 \mathrm{D}$ ). These findings strongly suggest that PKD1 has an inhibitory effect on OS cell invasion.

OS cell migration was significantly suppressed by PKD1 overexpression. Since cell migration is necessary at the initiation of tumor growth and metastases (25), we next evaluated the role of PKD1 on OS cell migration using in vitro scratch wound healing assays. At the time of wounding (at $0 \mathrm{~h}$ ), there was no apparent difference between cells that were transfected with HA.PKD1 or PKD1-siRNA or their controls (Fig. 3). After $16 \mathrm{~h}$ of incubation, in all three OS cell lines the wound closure in HA.PKD1 transfected cells was significantly suppressed compared with that in the control ( ${ }^{*} \mathrm{P}<0.05$, Fig. 3A). Conversely, in PKD1siRNA transfected cells, the wound closure was dramatically increased when compared with control ( ${ }^{*} \mathrm{P}<0.05$, Fig. 3B).

PKD1 expression negatively regulated MMP2 expression. It is known that OS cells produce MMPs to facilitate cell invasion and migration (20), and according to previous reports, MMP2 is strongly related with OS cell invasion and migration $(20,26)$. Therefore, we examined the effect of PKD1 expression on MMP2 regulation. We evaluated the expression of MMP2 in plasmid- or siRNA-transfected OS cells using qRT-PCR (Fig. 4A and C), and immunoblot analyses (Fig. 4B and D). MMP2 mRNA expression was significantly decreased by PKD1 overexpression $\left({ }^{*} \mathrm{P}<0.05\right.$, Fig. 4A), and was increased by siRNA knockdown of PKD1, 
A
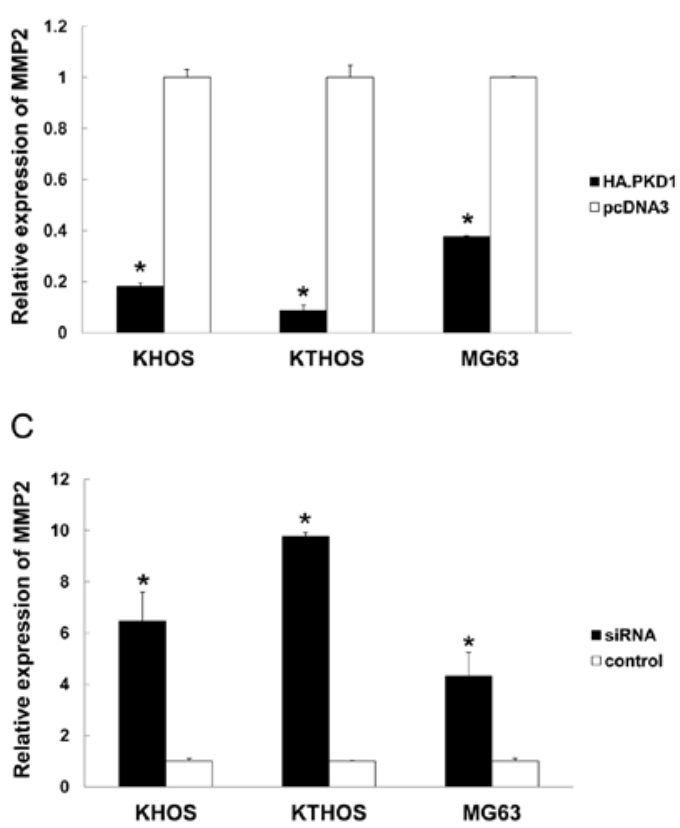

B
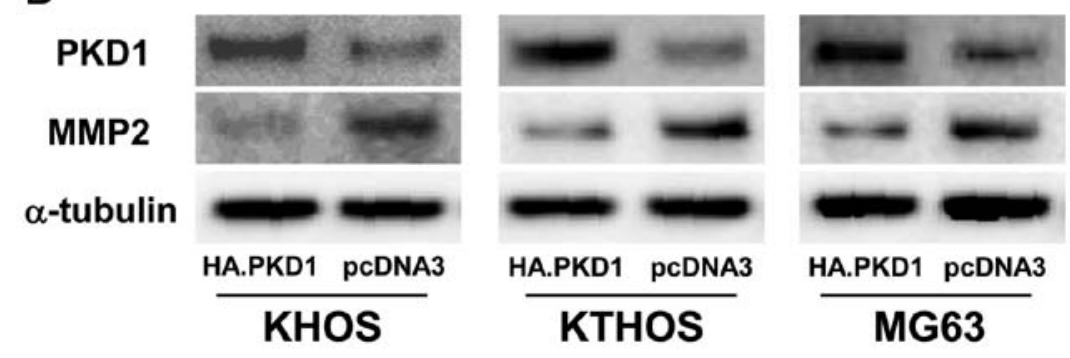

D

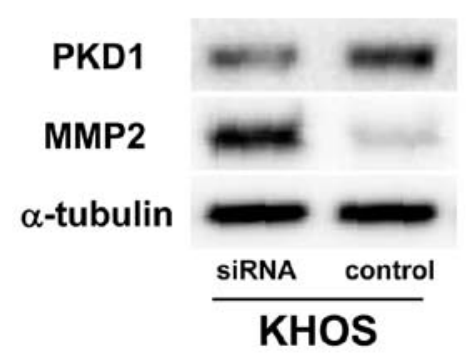

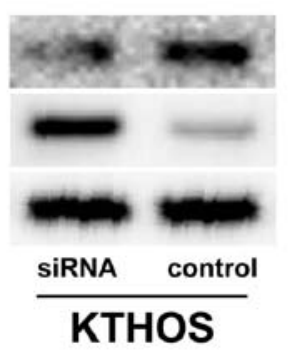

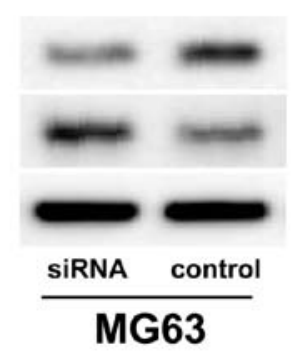

Figure 4. Expression of MMP2 in OS cells transfected with either HA.PKD1 plasmid or PKD1-siRNA. In PKD1-overexpressing OS cells, MMP2 expression was significantly decreased at both the mRNA $\left({ }^{*} \mathrm{P}<0.05\right)(\mathrm{A})$ and protein level (B) compared with control cells. Conversely, expression was increased by PKD1 knockdown at both the mRNA $\left({ }^{*} \mathrm{P}<0.05\right)(\mathrm{C})$ and protein level (D).

conversely ( $\mathrm{P}<0.05$, Fig. 4C). Immunoblot analyses revealed that significant suppression of MMP2 protein expression was observed in PKD1-overexpressing OS cells (Fig. 4B), and PKD1 knockdown strongly increased MMP2 protein expression (Fig. 4D). These results suggest that PKD1 expression negatively regulates MMP2 expression, and that OS cell migration and invasion properties might be related to PKD1-regulated MMP2 expression.

OS cell proliferation was decreased by PKDI overexpression. We also assessed the proliferative ability of OS cells that were transfected with either HA.PKD1 or PKD1-siRNA. In all cell lines, proliferation was significantly reduced by transfection of HA.PKD1 ( ${ }^{*} \mathrm{P}<0.05$, Fig. 5A), and was strongly increased by siRNA knockdown of PKD1 ( ${ }^{*} \mathrm{P}<0.05$, Fig. 5B). The results were corroborated by PCNA mRNA expression, a cell proliferation marker, using qRT-PCR (Fig. 5). The findings suggest that PKD1 has an inhibitory effect on OS cell proliferation.

Overexpression of PKDI showed a significant inhibitory effect on in vivo OS cell proliferation. Finally, we evaluated the effect of PKD1 overexpression on in vivo OS cell proliferation. We performed transfection of MG63 cells with either HA.PKD1 plasmid or control plasmid (pcDNA3), and each group of transfected cells were inoculated subcutaneously to the dorsal area of nude mice. Tumor growth formed by HA.PKD1 transfected cells was significantly suppressed compared with control cells ( ${ }^{*} \mathrm{P}<0.05$, Fig. 6A). qRT-PCR and immunohistochemical analysis of the tumors revealed that PKD1 expression was increased in tumors that were formed by HA.PKD1 transfected cells ( ${ }^{*} \mathrm{P}<0.05$, Fig. 6B-D). The results indicate that PKD1 overexpression decreases in vivo OS cell proliferation.

\section{Discussion}

Protein kinases are of particular interest in the treatment of human diseases because of their enzymatic activities and susceptibilities to successful therapeutic targeting (27). Since it was established that PKC is a high-affinity intracellular receptor for the phorbol-ester tumor promoters (28), many studies of PKC isozymes have been performed to elucidate their roles in cancer signal transduction pathways. Several studies have focused on PKC isozymes in musculoskeletal tumors (29-32). Kang et al reported that PKC $\theta$ expression is increased in Ewing's sarcoma (31), while Tirado et al demonstrated that PKC $\alpha$ phosphorylation promotes resistance to chemotherapy-induced apoptosis in Ewing's sarcoma cells (32). We have previously demonstrated that PKC412, a selective inhibitor of classical PKC, has an inhibitory effect on cell proliferation in human osteosarcoma and MFH cell lines by reducing PKC $\alpha$ activity (29). Our recent study revealed that $\mathrm{PKC} \delta$ has a negative effect on tumorigenesis and/or acts as a pro-apoptotic kinase in human MFH cells (30).

PKD1, a PKC-related kinase, has been shown to play important roles in multiple cellular functions, including cell proliferation, migration, invasion, DNA synthesis, apoptosis, oxidative stress response, immune response and other intracellular signal transduction pathways (27). Previous studies revealed that PKD1 is dysregulated in several cancer types (14-16). Kim et al analyzed PKD1 expression levels in primary gastric tumors and in gastric cancer cell lines, and revealed that the expression was decreased due to hypermethylation of the PKD1 promoter region (15). Analysis of invasive human breast tumors has revealed that PKD1 expression is downregulated in infiltrating ductal carcinoma compared to normal breast tissues (16), and androgen-independent human prostate cancer also showed 
A

KHOS

KTHOS

MG63
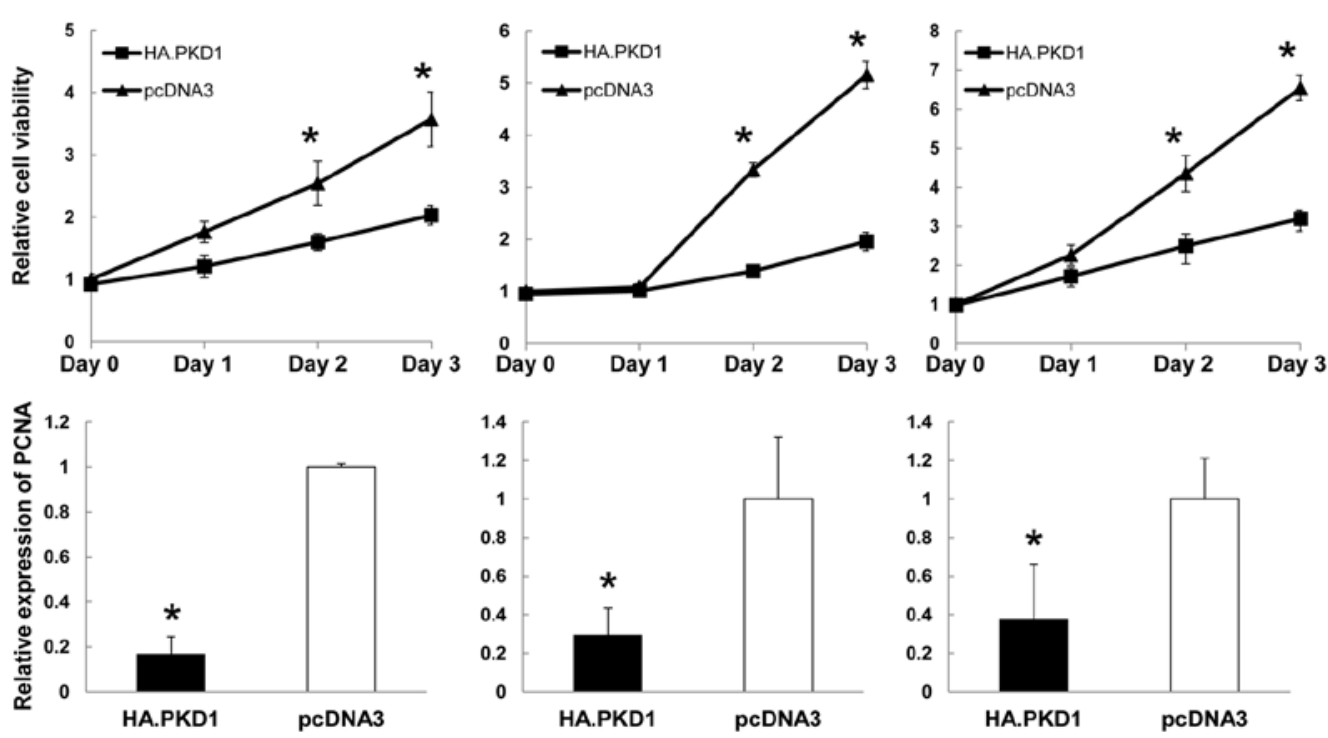

B

KHOS

KTHOS

MG63
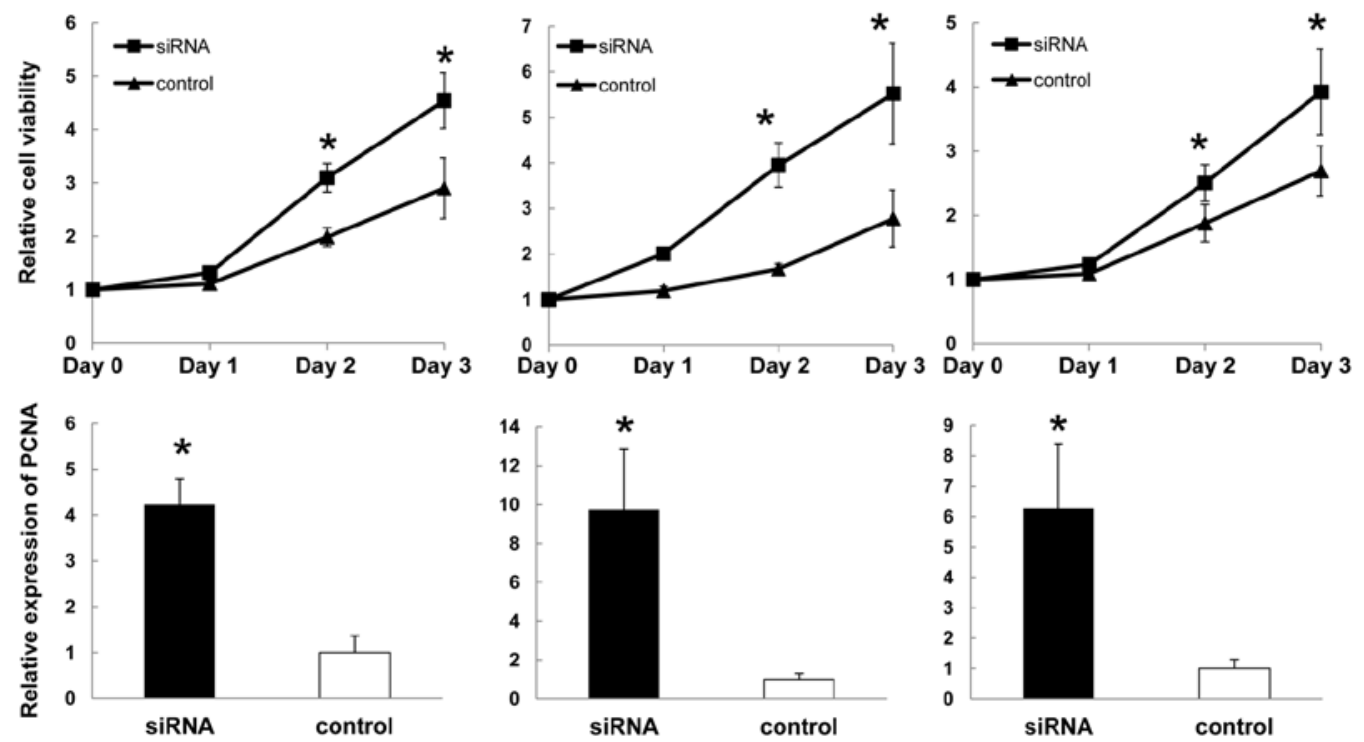

Figure 5. Cell proliferation assays and PCNA expression in PKD1 plasmid- or siRNA-transfected OS cells. Cell proliferation was significantly reduced by overexpression of PKD1 ("P<0.05) (A), and was increased by PKD1 knockdown ( $\mathrm{P}<0.05)(\mathrm{B})$. The results corroborated with mRNA expression of PCNA under the same conditions $(\mathrm{P}<0.05)(\mathrm{A}$ and $\mathrm{B})$.

reduced PKD1 expression (14). Therefore, PKD1 has recently received a considerable attention as a potential therapeutic target for human malignancies. On the other hand, studies focused on PKD1 in human pancreatic ductal adenocarcinoma and basal cell carcinoma have shown an opposing role for PKD1 $(33,34)$. There is even a report that PKD1 expression level was elevated in human prostate carcinoma tissues compared to normal prostate epithelial tissues (35). However, the role of PKD1 in musculoskeletal tumors is still unclear. We speculated that PKD1 is related with musculoskeletal tumorigenesis similarly to other cancers, and examined the expression of PKD1 in musculo- skeletal tumors. We found that mRNA expression of PKD1 in OS samples was significantly lower than that in benign schwannoma samples.

OS is a malignant bone-forming mesenchymal tumor and represents the most common primary tumor of the bone $(1,36)$. In aggressive growth, the worst problem of OS is their high propensity to form pulmonary metastases (37), and metastatic lesions have already developed in some cases by the time diagnosis has been made. Over the past two decades, progress in modern chemotherapy has improved the prognosis of patients with the disease (37), however the prognosis depends on the existence of 
A

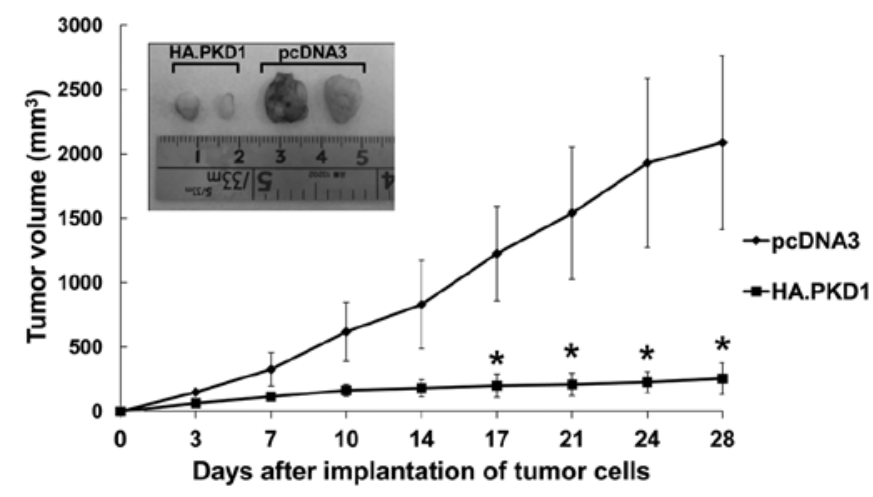

C

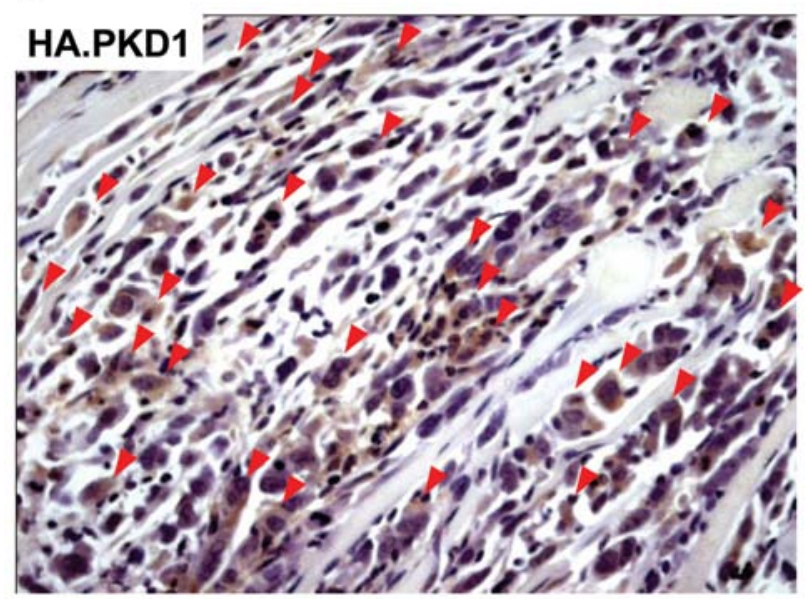

B

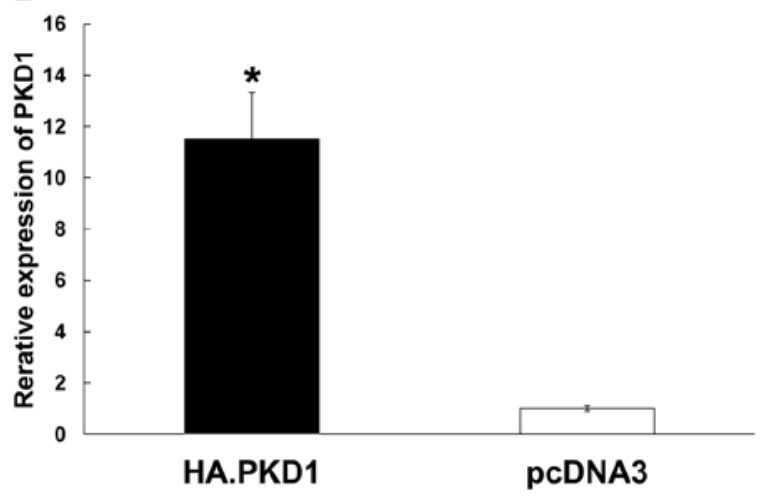

D

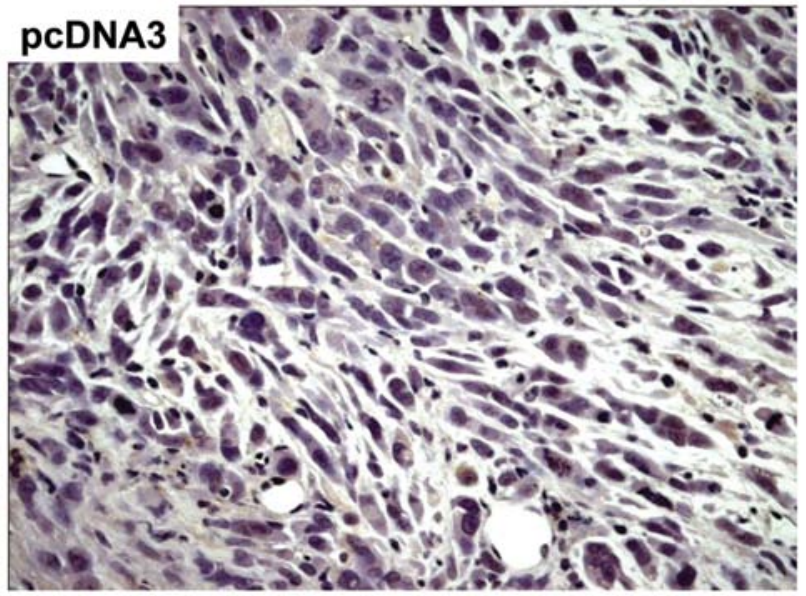

Figure 6. Effect of PKD1 overexpression on in vivo OS cell proliferation. We performed transfection of MG63 cells with either HA.PKD1 or control plasmid (pcDNA3), and subcutaneous inoculation of transfected cells into the dorsal region of nude mice ( $\mathrm{n}=12)$. There was no significant difference in body weight between the two groups (data not shown) during the experiments. The volume of tumors in HA.PKD1 transfected group was significantly lower than that in control group after 17 days of inoculation $\left({ }^{*} \mathrm{P}<0.05\right)(\mathrm{A})$. qRT-PCR showed that PKD1 expression in tumors in the HA.PKD1 transfected group was significantly increased compared with control ( $\mathrm{P}<0.05)(\mathrm{B})$, and increased PKD1 expression in tumors were also detected by immunohistochemical staining $(\mathrm{C}$ and $\mathrm{D})$. Red arrows indicate PKD1-overexpressing cells (C).

metastatic lesions rather than the primary tumor (38). Metastasis is considered to be the final step in the process of multistep tumorigenesis of malignant tumors (39), and is a complex process that is dependent on the capacity of a tumor cell capacity to invade, migrate and proliferate $(25,40)$. Previous studies reported that changes in PKC activity affects the malignant phenotypic characteristics in various cancers through cell signaling cascades (41). Jaggi et al reported that decreased PKD1 activity contributes to the malignant phenotype in androgen-independent prostate cancer because PKD1 is involved in multiple malignant processes (14), and PKD1 is also reported to be an inhibitor of breast cancer cell invasion (16). Therefore, we speculated that decreased PKD1 contributes to the malignant phenotype in OS, and revealed that PKD1 expression in metastatic OS samples was significantly lower than that in non-metastatic OS samples. Our findings suggest a significant correlation between PKD1 and metastatic potential of OS.

We next performed transfection of OS cell lines with PKD1overexpressing plasmid or PKD1-siRNA to evaluate the direct effects of PKD1 expression on OS cell invasion, migration and proliferation in vitro. We demonstrated that PKD1 overexpression significantly inhibited OS cell invasion and migration with suppression of MMP2 expression, and reduced cell proliferation. Conversely, siRNA knockdown of PKD1 dramatically increased cell invasion, migration, elevated the expression of MMP2, and enhanced proliferation of OS cells. These findings suggest that reduced expression of PKD1 is associated with oncogenic events in OS cells, consistent with previous reports $(11,15,16,42,43)$.

Migration is crucial for cancer cell spread (44), and can be classified into collective and single cell migration. Single migration consists of two alternative mechanisms of migration, namely amoeboid and mesenchymal migration (45). According to a report by Yui et al (46), acquisition of the mesenchymal mode of migration is critical in pulmonary metastasis of OS, which is mesenchymal in origin. This report also demonstrated that Rho-associated kinase (ROCK) inhibitor increases OS cell migration with induction of migration mode from amoeboid to mesenchymal (46). PKD1 is activated by RhoA via ROCK, which has a serine/threonine domain (47), and Matsumoto et al reported that highly activated Rho-ROCK pathway inhibited OS cell invasion and migration with decreasing MMP2 activity (48). Therefore, a possible mechanism of activation of OS cell migration is through Rho-ROCK-PKD1 pathway signaling. 
In breast cancer, tumor cells invade surrounding tissues by breaking through the basal membrane using invadopodia, which participates in proteolytic matrix degradation (6), and PKD1 forms a complex with cortactin and paxillin, both of which are associated with invadopodia membranes (6). Enomoto et al reported that, in OS cells, suppressed expression of either Wnt5a or Ror2 inhibits cell invasiveness accompanying decreased invadopodia formation (49). One of the most important steps in the metastatic process is the degradation of extracellular matrix (ECM), which allows a cell to invade and migrate to surrounding area (19), and MMPs have been shown to play a crucial role in helping cancer cells invade through the ECM and form metastatic lesions (50). Previous studies demonstrated that highly invasive OS expresses greater levels of MMP2 than poorly invasive OS (20), and that OS cell invasion and migration are decreased by reduced MMP2 expression (26). From these reports, we hypothesized that MMP2 may be a key target for PKD1-related migration and invasion. Then, we examined the direct effect of PKD1 expression on MMP2 expression in OS cell lines, and found that MMP2 expression was decreased by PKD1 overexpression, and conversely increased by PKD1 knockdown. Consistent with previous reports $(16,43)$, our data strongly suggest that overexpression of PKD1 may prevent OS cell migration and invasion by reducing the expression of MMP2. Furthermore, we examined the effect of PKD1 overexpression on in vivo OS cell growth, and revealed that tumor growth formed by PKD1-overexpressing OS cells was significantly decreased compared with control. The result indicates that targeting PKD1 is an effective tool for human OS in the clinical setting.

Taken together, these data are consistent with the possibility that PKD1 plays an important functional role in preventing tumorigenesis in human OS in vitro and in vivo. To our knowledge, this is the first report of the anti-tumoral effect of PKD1 on cell invasion, migration and proliferation in human OS. In conclusion, our findings strongly indicate that PKD1 may be a promising target for treatment and as a biomarker of progression of human OS.

\section{Acknowledgments}

We thank Takeshi Ueha, Minako Nagata, Maya Yasuda, and Kyoko Tanaka for expert technical assistance.

\section{References}

1. Meyers PA, Heller G, Healey J, et al: Chemotherapy for nonmetastatic osteogenic sarcoma: the Memorial Sloan-Kettering experience. J Clin Oncol 10: 5-15, 1992.

2. Johannes FJ, Prestle J, Dieterich S, Oberhagemann P, Link G and Pfizenmaier K: Characterization of activators and inhibitors of protein kinase C mu. Eur J Biochem 227: 303-307, 1995.

3. Johannes FJ, Prestle J, Eis S, Oberhagemann P and Pfizenmaier K: $\mathrm{PKCu}$ is a novel, atypical member of the protein kinase $\mathrm{C}$ family. J Biol Chem 269: 6140-6148, 1994.

4. Lavalle CR, George KM, Sharlow ER, Lazo JS, Wipf P and Wang QJ: Protein kinase D as a potential new target for cancer therapy. Biochim Biophys Acta 1806: 183-192, 2010.

5. Van Lint J, Rykx A, Maeda Y, et al: Protein kinase D: an intracellular traffic regulator on the move. Trends Cell Biol 12: 193-200, 2002.

6. Bowden ET, Barth M, Thomas D, Glazer RI and Mueller SC: An invasion-related complex of cortactin, paxillin and PKCmu associates with invadopodia at sites of extracellular matrix degradation. Oncogene 18: 4440-4449, 1999.
7. Rozengurt E, Rey $\mathrm{O}$ and Waldron RT: Protein kinase D signaling. J Biol Chem 280: 13205-13208, 2005.

8. Storz P and Toker A: Protein kinase D mediates a stress-induced NF-kappaB activation and survival pathway. EMBO J 22: 109-120, 2003.

9. Yang JM, Chin KV and Hait WN: Interaction of P-glycoprotein with protein kinase $\mathrm{C}$ in human multidrug resistant carcinoma cells. Cancer Res 56: 3490-3494, 1996.

10. Henttu P and Vihko P: The protein kinase $C$ activator, phorbol ester, elicits disparate functional responses in androgen-sensitive and androgen-independent human prostatic cancer cells. Biochem Biophys Res Commun 244: 167-171, 1998.

11. Jaggi M, Rao PS, Smith DJ, et al: E-cadherin phosphorylation by protein kinase D1/protein kinase $C\{\mathrm{mu}\}$ is associated with altered cellular aggregation and motility in prostate cancer. Cancer Res 65: 483-492, 2005.

12. Song J, Li J, Lulla A, Evers BM and Chung DH: Protein kinase D protects against oxidative stress-induced intestinal epithelial cell injury via Rho/ROK/PKC-delta pathway activation. Am J Physiol Cell Physiol 290: C1469-C1476, 2006.

13. Simpson KJ, Dugan AS and Mercurio AM: Functional analysis of the contribution of RhoA and RhoC GTPases to invasive breast carcinoma. Cancer Res 64: 8694-8701, 2004.

14. Jaggi M, Rao PS, Smith DJ, Hemstreet GP and Balaji KC: Protein kinase $\mathrm{C} \mathrm{mu}$ is down-regulated in androgen-independent prostate cancer. Biochem Biophys Res Commun 307: 254-260, 2003.

15. Kim M, Jang HR, Kim JH, et al: Epigenetic inactivation of protein kinase D1 in gastric cancer and its role in gastric cancer cell migration and invasion. Carcinogenesis 29: 629-637, 2008.

16. Eiseler T, Doppler H, Yan IK, Goodison S and Storz P: Protein kinase D1 regulates matrix metalloproteinase expression and inhibits breast cancer cell invasion. Breast Cancer Res 11: R13, 2009.

17. Choong PF: The molecular basis of skeletal metastases. Clin Orthop Relat Res 415 (Suppl): S19-S31, 2003.

18. Hidalgo $\mathrm{M}$ and Eckhardt SG: Development of matrix metalloproteinase inhibitors in cancer therapy. J Natl Cancer Inst 93: 178-193, 2001.

19. Egeblad $M$ and Werb Z: New functions for the matrix metalloproteinases in cancer progression. Nat Rev Cancer 2: 161-174, 2002.

20. Bjornland K, Flatmark K, Pettersen S, Aaasen AO, Fodstad O and Maelandsmo GM: Matrix metalloproteinases participate in osteosarcoma invasion. J Surg Res 127: 151-156, 2005.

21. Hitora T, Yamamoto T, Akisue T, et al: Establishment and characterization of a KIT-positive and stem cell factor-producing cell line, KTHOS, derived from human osteosarcoma. Pathol Int 55: 41-47, 2005.

22. Albini A: Tumor and endothelial cell invasion of basement membranes. The matrigel chemoinvasion assay as a tool for dissecting molecular mechanisms. Pathol Oncol Res 4: 230-241, 1998.

23. Liang CC, Park AY and Guan JL: In vitro scratch assay: a convenient and inexpensive method for analysis of cell migration in vitro. Nat Protoc 2: 329-333, 2007.

24. Okada Y, Akisue T, Hara H, et al: The effect of bevacizumab on tumour growth of malignant fibrous histiocytoma in an animal model. Anticancer Res 30: 3391-3395, 2010.

25. Woodhouse EC, Chuaqui RF and Liotta LA: General mechanisms of metastasis. Cancer 80: 1529-1537, 1997.

26. Fromigue $\mathrm{O}$, Hamidouche $\mathrm{Z}$ and Marie PJ: Blockade of the RhoA-JNK-c-Jun-MMP2 cascade by atorvastatin reduces osteosarcoma cell invasion. J Biol Chem 283: 30549-30556, 2008.

27. Jaggi M, Du C, Zhang W and Balaji KC: Protein kinase D1: a protein of emerging translational interest. Front Biosci 12: 3757-3767, 2007.

28. Kikkawa U, Takai Y, Tanaka Y, Miyake R and Nishizuka Y: Protein kinase $\mathrm{C}$ as a possible receptor protein of tumor-promoting phorbol esters. J Biol Chem 258: 11442-11445, 1983.

29. Kawamoto T, Akisue T, Kishimoto K, et al: Inhibition of PKCalpha activation in human bone and soft tissue sarcoma cells by the selective PKC inhibitor PKC412. Anticancer Res 28: 825-832, 2008.

30. Fukase N, Kawamoto T, Kishimoto K, et al: Protein kinase Cdelta in tumorigenesis of human malignant fibrous histiocytoma. Oncol Rep 26: 1221-1226, 2011.

31. Kang GH, Kim KM, Park CK and Kang DY: PKC-theta expression in Ewing sarcoma/primitive neuroectodermal tumour and malignant peripheral nerve sheath tumour. Histopathology 55: 368-369, 2009. 
32. Tirado OM, MacCarthy CM, Fatima N, Villar J, Mateo-Lozano S and Notario V: Caveolin-1 promotes resistance to chemotherapyinduced apoptosis in Ewing's sarcoma cells by modulating PKCalpha phosphorylation. Int J Cancer 126: 426-436, 2010.

33. Trauzold A, Schmiedel S, Sipos B, et al: PKCmu prevents CD95mediated apoptosis and enhances proliferation in pancreatic tumour cells. Oncogene 22: 8939-8947, 2003.

34. Ristich VL, Bowman PH, Dodd ME and Bollag WB: Protein kinase D distribution in normal human epidermis, basal cell carcinoma and psoriasis. Br J Dermatol 154: 586-593, 2006.

35. Chen J, Deng F, Singh SV and Wang QJ: Protein kinase D3 (PKD3) contributes to prostate cancer cell growth and survival through a PKCepsilon/PKD3 pathway downstream of Akt and ERK 1/2. Cancer Res 68: 3844-3853, 2008.

36. Forest M: Histologic classification of bone tumors (WHO, 1993). Ann Pathol 15: 226-227, 1995.

37. Wunder JS, Bull SB, Aneliunas V, et al: MDR1 gene expression and outcome in osteosarcoma: a prospective, multicenter study. J Clin Oncol 18: 2685-2694, 2000.

38. Whelan JS: Osteosarcoma. Eur J Cancer 33: 1611-1619, 1997.

39. Hanahan D and Weinberg RA: The hallmarks of cancer. Cell 100: 57-70, 2000.

40. Van Noorden CJ: Proteases and protease inhibitors in cancer. Acta Histochem 100: 344-354, 1998.

41. Wu D and Terrian DM: Regulation of caveolin-1 expression and secretion by a protein kinase cepsilon signaling pathway in human prostate cancer cells. J Biol Chem 277: 40449-40455, 2002.

42. Syed V, Mak P, Du C and Balaji KC: Beta-catenin mediates alteration in cell proliferation, motility and invasion of prostate cancer cells by differential expression of E-cadherin and protein kinase D1. J Cell Biochem 104: 82-95, 2008.
43. Biswas MH, Du C, Zhang C, Straubhaar J, Languino LR and Balaji KC: Protein kinase D1 inhibits cell proliferation through matrix metalloproteinase-2 and matrix metalloproteinase-9 secretion in prostate cancer. Cancer Res 70: 2095-2104, 2010.

44. Gupta GP and Massague J: Cancer metastasis: building a framework. Cell 127: 679-695, 2006.

45. Friedl $P$ and Wolf K: Tumour-cell invasion and migration: diversity and escape mechanisms. Nat Rev Cancer 3: 362-374, 2003.

46. Yui Y, Itoh K, Yoshioka K, et al: Mesenchymal mode of migration participates in pulmonary metastasis of mouse osteosarcoma LM8. Clin Exp Metastasis 27: 619-630, 2010.

47. Cowell CF, Yan IK, Eiseler T, Leightner AC, Doppler H and Storz P: Loss of cell-cell contacts induces NF-kappaB via RhoAmediated activation of protein kinase D1. J Cell Biochem 106: 714-728, 2009.

48. Matsumoto Y, Tanaka K, Harimaya K, Nakatani F, Matsuda S and Iwamoto Y: Small GTP-binding protein, Rho, both increased and decreased cellular motility, activation of matrix metalloproteinase 2 and invasion of human osteosarcoma cells. Jpn J Cancer Res 92: 429-438, 2001.

49. Enomoto M, Hayakawa S, Itsukushima S, et al: Autonomous regulation of osteosarcoma cell invasiveness by Wnt5a/Ror2 signaling. Oncogene 28: 3197-3208, 2009.

50. Liotta LA and Stetler-Stevenson WG: Metalloproteinases and cancer invasion. Semin Cancer Biol 1: 99-106, 1990. 